\title{
A Formwork Layout Model based on Genetic Algorithm
}

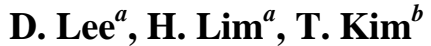 \\ $*$ H. Cho ${ }^{a}$ and K.I. Kang ${ }^{a}$ \\ ${ }^{a}$ School of Civil, Environmental and Architectural Engineering Korea University \\ Seoul, Republic of Korea \\ ${ }^{b}$ Research Institute of Engineering and Technology, College of Engineering, Korea University \\ Seoul, Republic of Korea \\ * Corresponding author (hhcho@ Korea.ac.kr)
}

\begin{abstract}
-
The recent increase in the number of irregularly shaped tall building has caused a continuous increase in the formwork costs. To reduce the cost of forms, modular forms are widely used since they are cheap and reusable. However, they cannot be applied around columns which are irregularly arranged. This study introduces a formwork layout model using a genetic algorithm to reduce formwork material costs. The proposed model seeks an optimum formwork layout to maximize the proportion of modular forms by arranging 12 types of modular forms with the columns, for which the positions and sizes of are changeable. From a casestudy, the model showed a decrease in the ratio of non-modular forms covering the area by about $11.9 \%$, and the rental cost of forms can be reduced by $10.4 \%$ compared to the previous heuristic method. The model can be used in the structural planning stage by a structural engineer to reduce the cost of formwork, and increase the efficiency of the column layout.
\end{abstract}

Keywords -

formwork layout; optimization; genetic algorithm; tall building

\section{Introduction}

The recent increase in the number of irregularly shaped tall building has caused a continuous increase in the formwork costs (Kim et al 2012). The material cost of forms has especially increased, while the labour cost has been reduced by the application of systemized formwork methods, since the number of non-modular form covered areas was highly increased because of irregularities. As the material cost of forms depends on the number and type of forms, the layout of formwork has become a key factor determining the formwork $\operatorname{cost}($ Cha 2012).
However, the present formwork layout planning method has several problems. First, planning is carried out in highly limited conditions because the structure of the building has already been fixed. For fixed structure layout conditions, only a few inefficient formwork layouts can be used. Second, the current formwork layout planning is conducted by someone experienced in heuristic approaches. Since there are an infinite number of formwork layout alternatives in a floor, it is impossible to make a quantitative decision about which layout solution is better within a short time, especially in large-sized and freeform shaped panels.

This study suggests a model for formwork layout planning to reduce formwork cost using genetic algorithm. The proposed model finds an optimal formwork layout alternative which maximizes the ratio of modular form-covered area by adjusting the size and position of the columns. To demonstrate the benefits, a case-study is implemented and then the result is compared with the layout alternative by an expert.

\section{The layout planning of formwork}

Existing studies related to formwork were overly weighted on selection of the formwork method. They focused only on the constructability and work productivity of the formwork, regarding the layout planning which must precede the formwork as unimportant (Lee et al 2009; Huang et al 2004; Tam et al 2005).

However, through in-depth interviews with practitioners from manufacturers, formwork engineers and suppliers, we found that one of the main issues with horizontal formwork is the process for finding optimal formwork layout solutions with the least cost.

When conducting formwork, the cost depends on the number and the type of the forms used. Therefore, the cost can be analysed quantitatively with different formwork layout alternatives (according to how many 
or what kind of forms are used).

There are two types of aluminium forms: the modular form, which is standardized in shape, and the non-modular form, which is not. The non-modular form is not reusable, commonly making the cost about 1.5 2 times more expensive than modular forms. This means that the formwork cost can be minimized by arranging forms of standard sizes to cover as much of the given space as possible, i.e., by minimizing the use of special units.

The objective of previous formwork layout planning was to cover the area with as many modular forms as possible. An expert arranged the forms on the floor plan from a start point to the end, from the biggest to the smallest, based on experience. The modular forms (standard sizes of which are $300 \mathrm{~mm}, 400 \mathrm{~mm}, 450 \mathrm{~mm}$, and $600 \mathrm{~mm}$ in width by $900 \mathrm{~mm}, 1050 \mathrm{~mm}$, and $1200 \mathrm{~mm}$ in length) are preferentially arranged, and non-modular forms are used only in the case when a modular form cannot be arranged.

In addition, when the formwork engineer designs aluminium formworks, beam units like end beam (EB) and main beam (MB) are always used to do the work more easily and safely. The beams attach aluminium forms more tightly, and prevent any kind of deflection even if all the forms are not similar enough (in size or position). Therefore, when making a layout planning model, the combination of beams must be considered. See Fig 1.

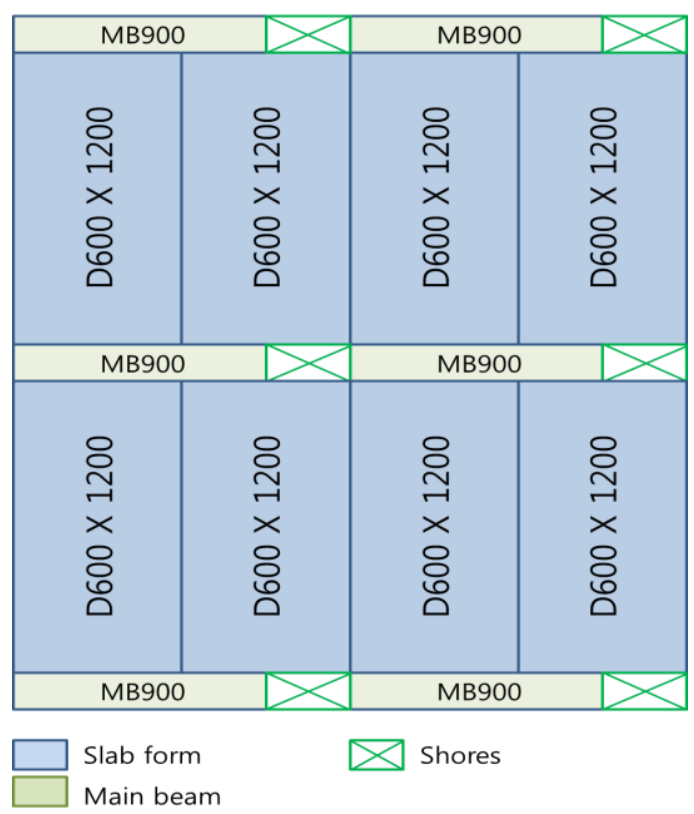

Figure 1. Main beams between the forms

According to the shape and the size of the floor plan, there are infinitely many kinds of formwork layout plans that can be made, so it is hard to find great layout alternatives within a short time. Especially, as the shape of buildings has become irregular and the layout of columns arranged unsystematically, the number of nonmodular forms has increased highly around the columns. This directly influences the rental cost of forms.

In short, previous formwork layout planning methods have two problems. One is that the formwork layout planning is conducted by someone with individual experience and intuition, making the planning lower-quality and cost-inefficient. The other is related with the formwork planning time, in that planning is carried out after finishing the structure layout planning. Since the layout planning is decided on a fixed structure layout, including locations of columns and walls, if the structural layout is complex, it cannot be helped but to use many non-modular forms.

Therefore, a computerized and automated layout planning model for optimum formwork layout which arranges forms at the planning stage without any limitation of the columns layouts would be greatly beneficial.

\section{Formwork layout model using genetic algorithm}

Through interview of experts who majored in the structure of buildings, we realized that changing the column layout (length, width, position etc.) within an allowable nominal area should be flexible at the planning stage. It would be critical to change the structure for the formwork, but considering that such change is required by the constructor and employer to reduce cost, limited adjustment can still be valuable enough

Formwork layout planning is conducted with lines since all of the forms are rectangular and are arranged in good order. This is similar to the two dimensional rectangle cutting stock problems. However, it is an NP(Non-Polynomial)-hard problem, because there are many variables. The position and size of the columns, forms and beams are changeable. There is no stiffness when planning the layout and the placement must be done one by one, which is why a heuristic algorithms was chosen instead of a mathematical method.

Like the previous formwork arrangement method, as many modular forms as possible were arranged to optimize the space, from the biggest form to the smallest one. Only when forms encountered columns, columns were deleted and the space was used as an optimization area. If there were columns in the same section in line, they were joined together and optimized at the same time.

We found that the beams that connect two different 
groups of forms always adhere to the form in the wider direction (Fig 1.), so they were counted as a part of the forms. Since the width of the beams is always $150 \mathrm{~mm}$, it was added to the width of the forms. The adjusted types of the modular forms are shown in Table 1.

Table 1. The types of the modular forms (12 in total)

\begin{tabular}{ccc}
\hline Height $(\mathrm{mm})$ & Width $(\mathrm{mm})$ & Area $\left(\mathrm{m}^{2}\right)$ \\
\hline 300 & 1050 & 0.315 \\
300 & 1200 & 0.36 \\
300 & 1350 & 0.405 \\
400 & 1050 & 0.42 \\
400 & 1200 & 0.48 \\
400 & 1350 & 0.54 \\
450 & 1050 & 0.4725 \\
450 & 1200 & 0.54 \\
450 & 1350 & 0.6075 \\
600 & 1050 & 0.63 \\
600 & 1200 & 0.72 \\
600 & 1350 & 0.81 \\
\hline
\end{tabular}

The formwork layout planning optimization process is as follows. First, input (1) the standard size of forms and their cost, (2) initial position of the columns as $\mathrm{x}, \mathrm{y}$ coordinates, (3) starting point and direction of arrangement. Second, arrange as many modular forms $(600 \times 1350)$ to the floor plan as possible. If there are any forms which are interfered with by columns, delete them and set the area as an optimization area. Third, grids are set to every optimization region at $50 \mathrm{~mm}$ intervals, considering the minimum unit of standard aluminium forms. After measuring the width and the height of the optimization area, the model will search for optimal solutions. The procedure shown in Fig 2., and the concept of the optimization is shown in Fig 3. respectively.

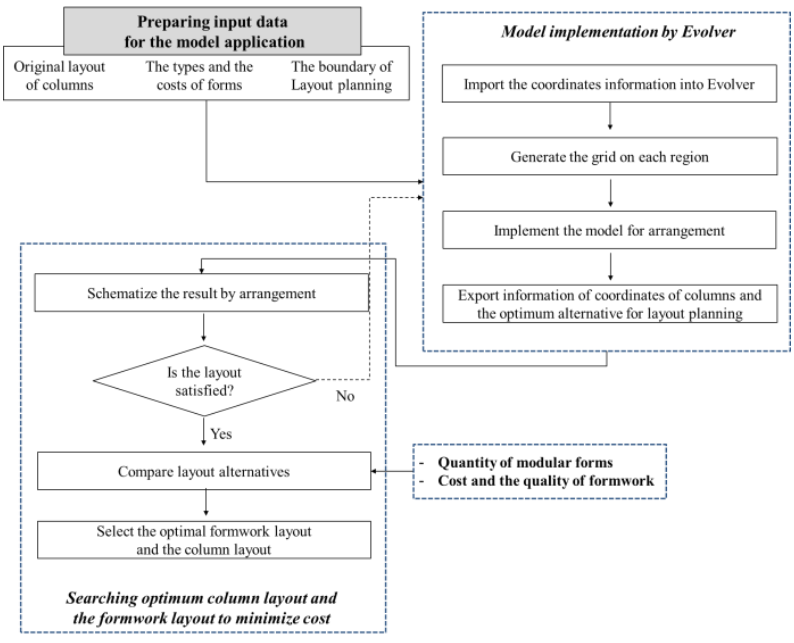

Figure 2. Procedure for formwork layout planning based on the optimizing model.

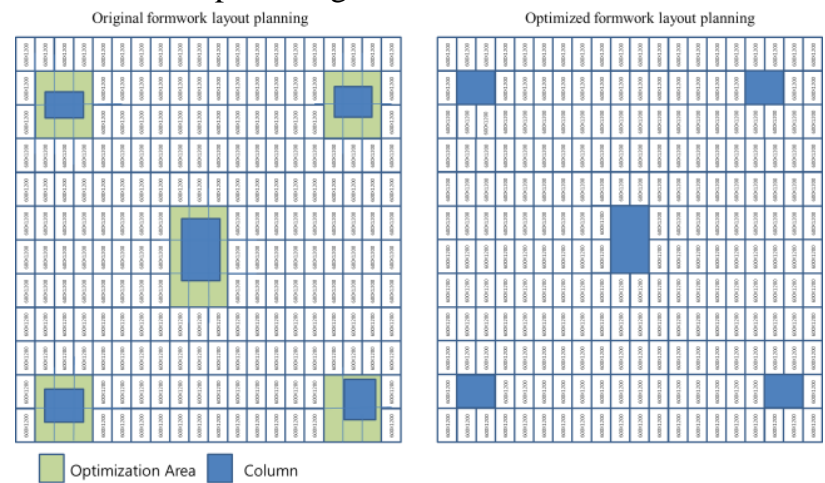

Figure 3. Schematic concept of movement of columns

A genetic algorithm is used in this process to obtain near optimal solutions.

The arrangement of forms and positioning of the columns is determined according to the gene sequences. The $\mathrm{x}$ axis gene sequence variable is $a_{i}$, and the $\mathrm{y}$ axis is $b_{i}$. The column layout variables $\mathrm{x}, \mathrm{y}, \mathrm{s}($ the coordinates and the sizes of columns) are defined. For example, the normal width size of forms, $1350 \mathrm{~mm}$, $1200 \mathrm{~mm}$ and $1050 \mathrm{~mm}$ can be allotted to the $\mathrm{x}$ axis array, and the height size of the forms, $300 \mathrm{~mm}, 400 \mathrm{~mm}$, $450 \mathrm{~mm}$, and $600 \mathrm{~mm}$ can be allotted to the y axis array independently. In the column layout array, any kind of $(\mathrm{x}, \mathrm{y})$ coordinate in the optimization area and the column's size can be allotted within $50 \mathrm{~mm}$. The gene sequences are shown in Fig 4.

\begin{tabular}{c|c|c|c|c|c|c|}
\cline { 2 - 7 } $\boldsymbol{X}_{\text {'axis array }}$ & $\boldsymbol{a}_{\mathbf{1}}$ & $\boldsymbol{a}_{2}$ & $\cdots$ & $\boldsymbol{a}_{\boldsymbol{k}}$ & $\cdots$ & $\boldsymbol{a}_{\boldsymbol{n}}$ \\
\cline { 2 - 7 } $\boldsymbol{Y}^{\prime}$ axis array & $\boldsymbol{b}_{1}$ & $\boldsymbol{b}_{2}$ & $\ldots$ & $\boldsymbol{b}_{\boldsymbol{k}}$ & $\ldots$ & $\boldsymbol{b}_{\boldsymbol{n}}$ \\
\cline { 2 - 7 } Column layout & $x_{1}, y_{1}, s_{1}$ & $x_{2}, y_{2}, s_{2}$ & $\ldots$ & $x_{k}, y_{k}, s_{k}$ & $\ldots$ & $x_{n}, y_{n}, s_{n}$ \\
\cline { 2 - 7 }
\end{tabular}

Figure 4. Gene sequences for formwork layout

Once the three gene arrays are allotted with numbers, a layout alternative is made. Fig 5, 6, 7 .

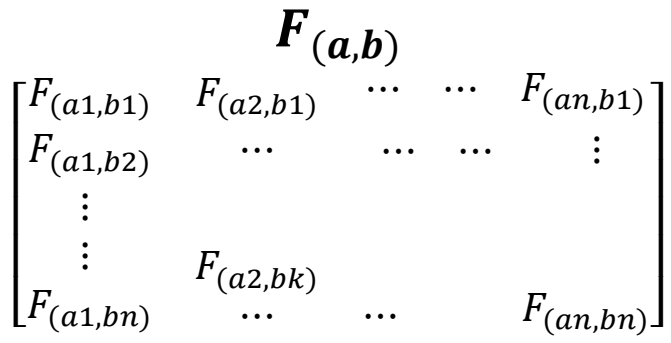

Figure 5. Formwork layout alternatives 


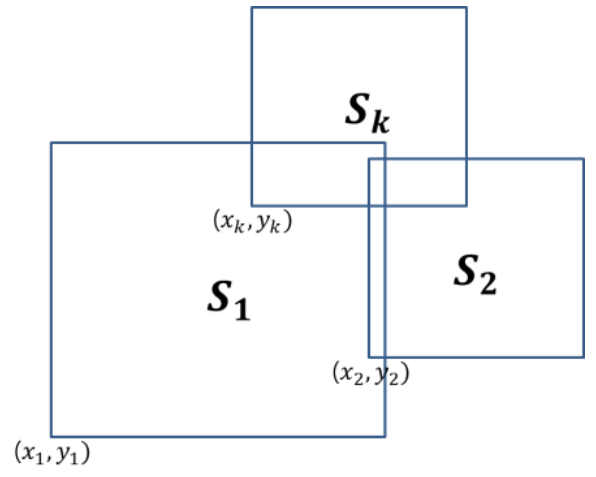

Figure 6. Column layout alternatives

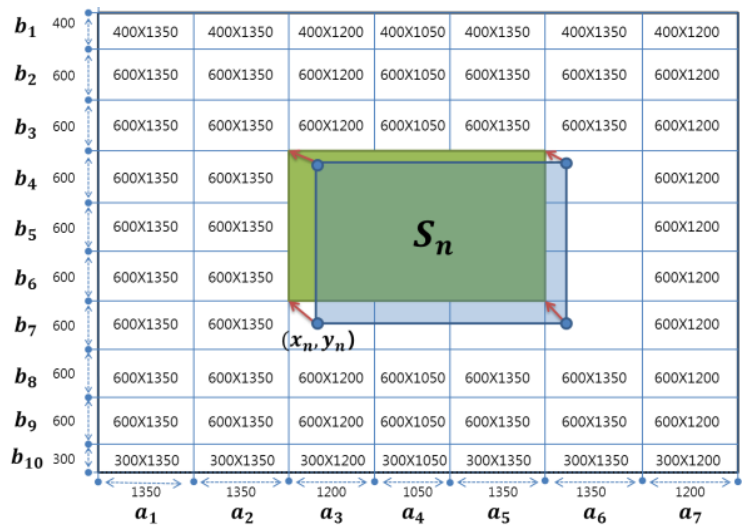

Figure 7. Schematic diagram of a formwork layout alternative

In this model, the objective function is represented by cost, the total formwork cost. By analysing the formwork layout matrix and the layout of the columns, the total cost can easily be calculated. Therefore, the total cost is a function of $\mathrm{a}, \mathrm{b}, \mathrm{x}, \mathrm{y}$, and $\mathrm{s}$, as follows:

$$
\text { Total cost }=\sum f\left(a_{i}, b_{i}, x_{i}, y_{i}, s_{i}\right)
$$

The model continuously changes these three sequences with a genetic algorithm, and compares the objective function until no better alternatives can be deduced.

All of formwork layout alternatives are deduced only using modular forms, and the forms are only regarded as non-modular when they are interfered with by obstacles. Arrangement of forms and columns is carried out by filling the gene array. In this process, the larger forms should be arranged first to reduce the total number of forms, and the summation of the total height of the forms should be perfectly fit to the height of the optimization area (same as the width).

In addition, the model user must deliberate the optimization boundary of columns and set the distance limitations, to secure safety and maintain the original design. Even if an alternative was verified as the most efficient result, it is possible that it may not be used because of safety or other factors such as design or law.

The cost of the forms are calculated with the type of the forms (modular or non-modular), and the covered area. If any forms encounter columns, the forms are estimated as non-modular forms.

\section{Case study}

\subsection{Case Description}

The model for formwork layout planning was applied to an actual project to verify its applicability and demonstrate the benefits. The project was a 50-story building, for which the floors are very irregular.

The computed layouts were compared with the layout obtained with the heuristic approach from a professional who had not only worked in formwork planning for 10 years, but had also participated in designing the aluminium forms. By comparing the modular form-covered area, the cost was quantitatively analysed. The detailed floor plan of the project is shown in Fig. 8

As can be seen, the shape is very irregular, with unsymmetrical column layouts.

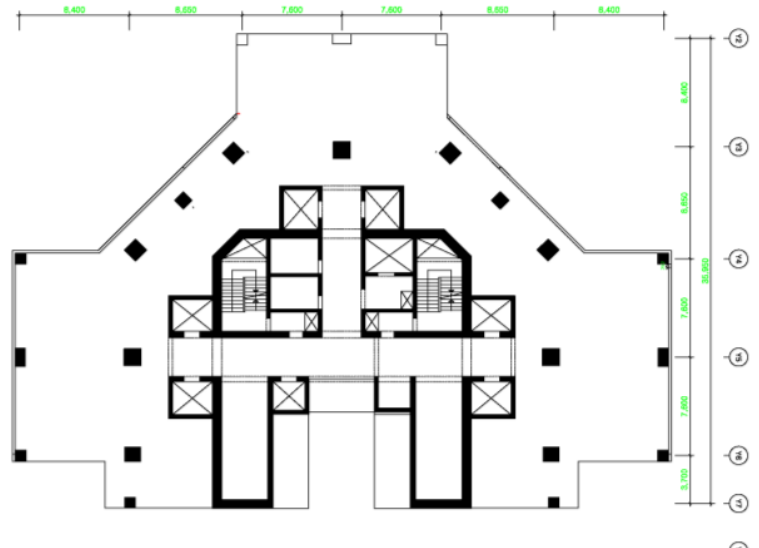

Figure 8. Floor plan of the case project

\subsection{Result and discussion}

The layout solutions from the model and the heuristic approach are shown in Fig 9. As can be seen, the solution generated by the proposed model used more modular forms than the heuristic solution.

In this analysis, we assumed the rental cost of the aluminium form to be US $\$ 3$ per meter square, and the cost of non-modular forms to be 2 times more expensive than the modular forms. The total area of the floor was originally $741.36 \mathrm{~m}^{2}$. The $50^{\text {th }}$ story was analysed. The 
detailed differences are shown in Table 2.

An accurate rental cost cannot be estimated, since it is determined based on the number of reuses, which could continuously be changed according to the floor plan and construction time.

Table 2. The results of formwork layout planning

\begin{tabular}{ccc}
\hline Items & $\begin{array}{c}(\mathrm{a}) \\
\text { by an expert }\end{array}$ & $\begin{array}{c}(\mathrm{b}) \\
\text { by the model }\end{array}$ \\
\hline $\begin{array}{c}\text { Non-modular form } \\
\text { covered area }\left(\mathrm{m}^{2}\right)\end{array}$ & 105.157 & 17.188 \\
$\begin{array}{c}\text { Rental cost of slab } \\
\text { formwork (US\$) }\end{array}$ & $126,977.6$ & $113,782.2$ \\
$\begin{array}{c}\text { Total time for } \\
\text { optimization }\end{array}$ & 4 hour & 30 min \\
\hline
\end{tabular}

From the results, about $88 \mathrm{~m}^{2}(11.9 \%)$ of the originally planned non-modular forms, comprised of at least 105 units, could be changed to modular forms. This reduced the rental cost by almost $\$ 10,195$ US. If the model is applied to bigger and higher buildings, the difference will increase. Also, the total time for optimization was reduced from 4 hours, by the expert, to only $30 \mathrm{~min}$, by the model. As can be seen in Fig 9 (a), non-modular forms are especially required around the columns, near the walls, and in the transition zone of the floor. On the other hand, it can be seen in Fig 9 (b) that the black marked area, non-modular forms, was considerably reduced. In addition, the array of the forms looks far more simple and easy to manage, consequently enabling minimization of the formwork cost.

In this study, we suggested a formwork layout model which can be used in the structural design stage to reduce formwork cost. The model assumed that columns can be moved within limited boundaries; however, this may sometimes be a critical issue to discuss, since columns are one of the main structural members and design factors, and their placement is also related with safety. So, consideration of safety should be made, depending on the circumstances.

This study has a major role in the cost consideration of formwork layout planning. A structural engineer who is aware of potential formwork costs has to consider the advantages of structural changes, and function at reduced costs. This advanced model can bring many benefits to owners, engineers and contractors.
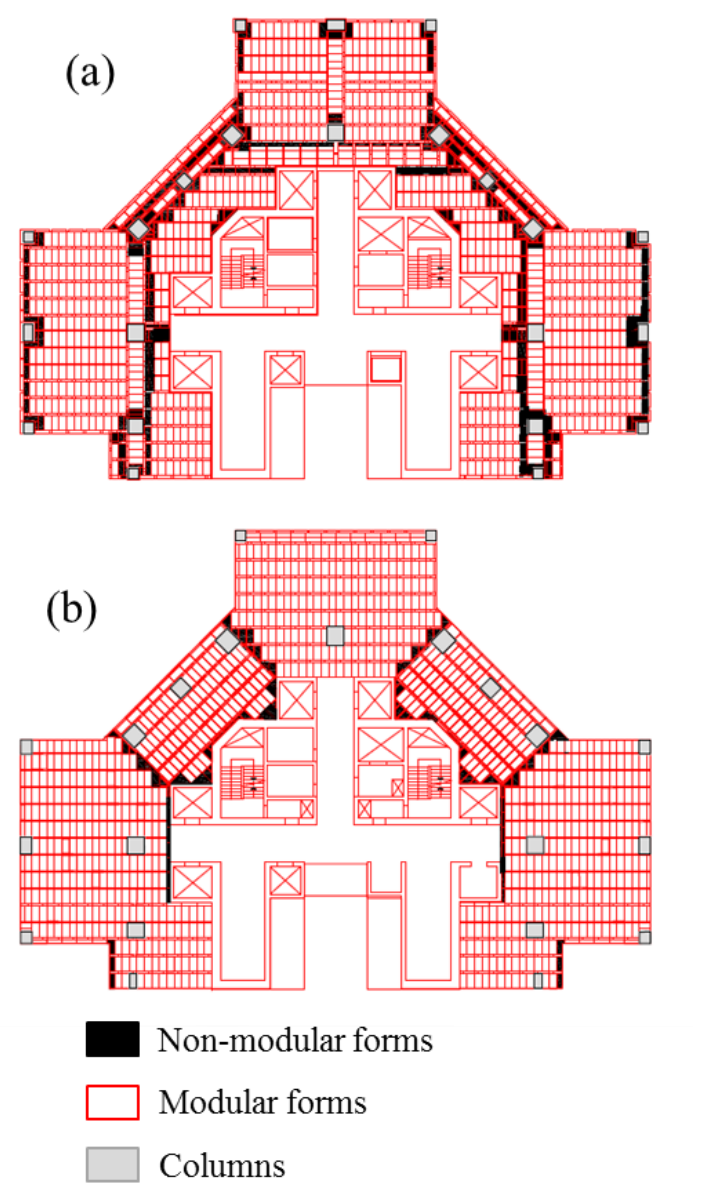

Figure 9. Comparison of layout results generated by (a) an expert, and (b) the model

\section{Conclusion}

We have proposed a new formwork layout planning model which is suitable for the irregularly shaped buildings.

The movement of columns within a limited region can be a great solution to reduce formwork cost without any losses. This model can be used in the structural planning stage by a structural engineer to reduce the cost of formwork, and increase the efficiency of the column layout. In addition, the computerized model can reduce the time required for layout planning, and enables flexibility in response even if there are any changes to the plan.

Through further studies, the consideration of changing the column design and the space distribution problem will be solved. In addition, another optimization algorithm will be studied to enhance the efficiency as well as to reduce the optimization time. 


\section{Acknowledgment}

This research was supported by a grant (Code\#09 R\&D A01) from the High-Tech Urban Development Program, funded by the Ministry of Land, Infrastructure and Transport Affairs of Korea. Special thanks to Mr. Seung-Bum Lim, who is an expert engineer in formwork design.

\section{Reference}

[1] Kim T., Lim H., Lee U. K., Cha M., Cho H., Kang K. I. Advanced formwork method integrated with a layout planning model for tall building construction. Canadian Journal of Civil Engineering. 39:1173-1183, 2012

[2] Kim T. Advanced system formwork and construction planning model for tall building construction. Doctorate thesis, Korea University, 2013

[3] Cha M. A Decision Support Model for Optimal Formwork Layout in High-rise Building Construction. Master's thesis, Korea University, 2012

[4] Huang T. and Hastak M. Simulation study on construction process of FRP bridge deck panels. Automation in Construction, 16(5), pp.620-631, 2007

[5] Lee C., Ham S., Lee G. The development automatic module for formwork layout using the BIM. ICCEM/ICCPM 2009. v.3, pp. 1266-1271, 2009

[6] C.M. T., T.K.L. T., T.C.T. L., K.K. C. Selection of vertical formwork system by probabilistic neural networks models. Constr. Manag. Econ. n.23, 245254,2005

[7] Lab R.H. Think Formwork - Reduce Costs. Online: http://www.structuremag.org/article.aspx?articleID $=423$, Accessed: 13/12/2013. 